\title{
LA EDUCACIÓN SUPERIOR COMO DEMANDA Y COMO RECURSO EN DISPUTA EN ESPACIOS INTER-ÉTNICOS. UN ANÁLISIS EN LAS COMUNIDADES WICHÍ DE SALTA.
}

\section{HIGHER EDUCATION AS A DEMAND AND AS A RESOURCE IN DISPUTE IN INTER- ETHNIC SPACES. AN ANALYSIS AT SALTA WICHÍ COMMUNITIES.}

\author{
Gloria Mancinelli ${ }^{1}$
}

Fecha de recepción: 24-06-2016

Fecha de aceptación y versión final: 12-09-2016

Resumen: El principal objetivo de este trabajo, consiste en producir algunas reflexiones en torno a demandas de educación superior que se vienen planteando en comunidades wichí de la provincia de Salta. Dichas demandas se inscriben en el conjunto de los reclamos educativos realizados por los movimientos sociales indígenas latinoamericanos, en pos del reconocimiento de las condiciones pluriétnicas y pluri-lingüísticas de nuestro país. Pero también deben comprenderse vinculadas con el impacto producido por las actuales políticas productivas y extractivas en los territorios donde se asientan estas poblaciones.

De esta forma, se analizan en primer lugar los reclamos de acceso a la educación superior en relación a los procesos de disputas y reconfiguraciones territoriales que atraviesan a estas poblaciones, como consecuencia del actual modelo extractivo que se desarrolla en la región nordeste de la provincia de Salta. En segundo lugar, comprendiendo que dicho contexto ha convocado al espacio a nuevos actores sociales (equipos de investigación y de extensión, ONGs, Fundaciones, agentes estatales) que desde sus prácticas reconfiguran el espacio social, analizamos de qué manera intervienen en la configuración de dichas demandas y contribuyen en la estructuración de prácticas que hacen posible condiciones de acceso a la educación superior en estas poblaciones.

Palabras clave: Pueblos Indígenas, Wichí, Educación Superior, Salta.

Abstract: The main objective of this paper is to produce some reflections about higher education demands that of-the wichí communities at the Salta's province. These demands are inscribed into the set educational claims proposing by Latin American indigenous social movements for the pluri-ethnic and pluri-linguistic conditions recognition of our country. In addition, it should be understood in relation to the impact of current economic extractive production that has been implemented in the territories where the populations live.

In this way, we analyze first the claims of access to higher education in relation to the processes of disputes and territorial reconfigurations that cross these populations because of the current extractive model developed in the northeast region of the Salta's province. Second, understanding that this context has summoned new social actors into that space (teams research and extension, NGOs, foundations, state agents) which from their participatory practices rewire the social space, we analyze in which way they are involved in the demands configuration, and how they contribuit in structuring practices that make possible the conditions for access to higher education in these populations.

\footnotetext{
${ }^{1}$ Profesora en Ciencias Antropológicas de la Universidad de Buenos Aires. Doctoranda de la Facultad de Filosofía y Letras y becaria doctoral de la nombrada universidad en el marco del Proyecto, "Impacto del conflicto por los recursos naturales del modelo extractivo en poblaciones étnicamente definidas", Sección de Antropología Social del Instituto de Ciencias Antropológicas de la Facultad de Filosofía y Letras de esa casa de estudios. Realiza su investigación con comunidades wichí del departamento de San Martín de la provincia de Salta. Su tema de investigación es sobre demandas de acceso a la Educación Superior en dichas comunidades y su vinculación con los procesos de re configuraciones territoriales que se han ido produciendo en la provincia. Correo electrónico: mancinelli.gloria@gmail.com.
} 
Key words: Indigenous Peoples, Wichí, Higher Education, Salta.

\section{Introducción}

Este artículo propone una reflexión en torno a la relación entre pueblos indígenas y educación superior, considerando tanto las demandas de acceso a las universidades nacionales tradicionales, como así también la apertura de espacios académicos de nivel terciario que atienden demandas de formación profesional dentro de las comunidades indígenas.

En estas experiencias se expresan complejidades vinculadas a procesos identitarios y de diversidad pluricultural, pluri-étnica y pluri-lingüística que se entrelazan en contextos de una profunda desigualdad social, económica y cultural. Estos contextos de diversidad y desigualdad que se han ido configurando en la dinámica histórica se combinan con nuevos procesos que agudizan aún más las condiciones de desigualdad económica al tiempo que proponen nuevas instancias de negociación entre diversos actores sociales y el estado. En esta arena se van (re)configurando los espacios socio territoriales y van emergiendo nuevas demandas y nuevas prácticas que involucran dinámicas de transmisión, apropiación, producción y reproducción cultural, e interpelan de forma particular a los espacios educativos formales.

Dentro de los trabajos que se han venido realizando en este campo para nuestro país, considero como punto de partida el trabajo de Paladino (2009), desde el cual es posible visualizar los indicadores educativos que se expresan en las poblaciones indígenas de Argentina, mostrando las diferencias respecto de los indicadores que presenta la población total nacional. El análisis de esta autora cobra relevancia para este trabajo, ya que ofrece información que se refiere no sólo al conjunto de la población indígena, donde se visualiza una significativa inequidad en el acceso a la escolaridad, especialmente en el nivel medio y superior, sino que también permite observar las diferencias que se expresan entre los diferentes pueblos. De acuerdo con este trabajo vemos que la población mapuche muestra una tasa del 15\% de jóvenes que transitan o ha transitado alguna experiencia en el nivel superior siendo el grupo con los mejores indicadores. En contraste la población wichí muestra una tasa del $0,5 \%$ de jóvenes que transitan o ha transitado alguna experiencia en el nivel superior encontrándose dentro de los grupos étnicos con los indicadores educativos más bajos en los últimos niveles de enseñanza.

Focalizando en la población wichí de la provincia de Salta, se recuperan los trabajos de Ossola $(2013,2015)$, los cuales pueden entenderse como de los primeros trabajos etnográficos producidos en nuestro país que indagan en la experiencia que realizan jóvenes wichí como estudiantes en la Universidad Nacional de Salta (UNSa). En dichos trabajos, la autora logra dar cuenta de los sentidos y las representaciones que asume la educación superior en contextos rurales, interculturales e interétnicos, destacando procesos de apropiación y de producción cultural que estos jóvenes realizan sobre los espacios institucionales de esta universidad. De acuerdo con esto, consideramos del trabajo de Ossola tanto la información producida a nivel poblacional 
sobre el pueblo wichí en su conjunto, como también el énfasis puesto en las dinámicas de apropiación y producción cultural que realizan los jóvenes universitarios wichí.

Por otra parte los trabajos Mato (2014), ofrecen uno de los primeros avances en la sistematización de las diferentes experiencias que se vienen realizando tanto en Argentina como en Latinoamérica, enfatizando el rol de los movimientos sociales indígenas en la apertura de este proceso, y remarcando el escaso impacto que aún vienen teniendo los marcos jurídicos que proclaman los derechos a la diversidad étnica, cultural y lingüística para lograr mayores avances en materia de inclusión e interculturalidad educativa en nivel superior. De este modo, los trabajos de Mato permiten acercarnos a la diversidad de experiencias que van conformando el complejo proceso de inclusión de indígenas en espacios de formación superior, las cuales no se muestran exentas de presentar continuidades y contradicciones vinculadas a las profundas matrices de los procesos de colonización en nuestro continente.

Asumiendo una línea historiográfica, y enfatizando en los procesos de (re) configuraciones territoriales que propone este artículo, recuperamos el trabajo de Rosso (2011) quien analiza la relación entre educación e indígenas, tomando el caso de la provincia de Chaco. Dicho trabajo considera los procesos de (re)configuraciones territoriales que sucedieron en esta provincia en torno a la conformación del estado nacional durante las primeras décadas del siglo pasado, donde se han ido estructurando matrices que pueden verse aún presentes en los espacios de educación formal de la provincia.

Articulando estas diferentes perspectivas, este trabajo se propone principalmente retomar la línea de indagación propuesta por Rosso (Ob. Cit.) para reflexionar en torno a los procesos de (re)configuraciones territoriales que acontecen actualmente en la provincia de Salta y la manera en que estos procesos interpelan particularmente a los espacios de formación superior (terciarios-universitarios) y sus vínculos con los pueblos indígenas de la región. De acuerdo con esto, centro el análisis en las experiencias que se realizan en dos comunidades wichí del departamento de San Martín de la provincia de Salta. En segundo lugar, y comprendiendo que estos procesos (re)configuran un espacio social/local, analizamos de qué forma la presencia de nuevos actores dentro de estas comunidades intervienen en la conformación de nuevas demandas educativas y contribuyen en la estructuración de prácticas y significados y hacen posible condiciones para mejorar el acceso a la educación superior de estas poblaciones. Finalmente sugiero algunas perspectivas de investigación que pueden ser consideradas a partir del análisis de las experiencias de las que da cuenta este trabajoi.

En términos metodológicos el trabajo se sustenta en una perspectiva cualitativa propia de la antropología social y cultural. De acuerdo con esto podemos mencionar como herramientas principales el trabajo de campo, la observación participante y las entrevistas en profundidad abiertas y semi-estructuradas. Respecto al trabajo de campo el mismo ha sido realizado durante el 2015 en diferentes espacios: la comunidad wichí Lotes Fiscales y la Comunidad wichí Misión Chaqueña, ambas pertenecientes al departamento de San Martín, ubicado al noreste de la provincia de Salta. A estos espacios se suma de manera articulada el trabajo de campo realizado el mismo año en la Universidad Nacional de Mar del Plata donde estudian actualmente 8 jóvenes wichí de las comunidades citadas. De esta manera, el trabajo de campo se 
configura como una práctica multi-situada donde se reconoce la "pluralidad de lugares" en los que se ve involucrada la práctica etnográfica contemporánea (Marcus, 2001). Las entrevistas fueron producidas con jóvenes universitarios y jóvenes que participan de alguna experiencia vinculada a la formación profesional dentro de las comunidades. A estas se suman entrevistas informales realizadas a directores y docentes e investigadores de proyectos de extensión universitaria involucrados en actividades de transferencia para estas mismas comunidades.

\section{Procesos territoriales en el noreste de la provincia de Salta y demandas educativas}

Las comunidades wichí consideradas para la investigación se encuentran emplazadas en el departamento de San Martin, municipio de Embarcación, en la provincia de Salta. Este municipio se ubica en la zona noreste de la provincia, y conforma parte de lo que se conoce como la región del chaco salteño caracterizada por sus recursos naturales, su biodiversidad y la extensión de sus bosques.

En esta región, especialmente a partir de los '90 puede observarse lo que se define como un desplazamiento de la frontera agropecuaria (Trinchero, Campos Muñoz, Valverde, 2014), donde la actividad agrícola a gran escala intensificó su producción, principalmente la que refiere al monocultivo de oleaginosa. En esta nueva expansión de la frontera agraria, basada hoy en la soja, se fue avanzando sobre territorios indígenas y criollos, produciéndose de esta forma una redefinición del territorio y de las condiciones de vida de estas poblaciones (Schmidt, 2010: 2). Este proceso impactó negativamente en las economías domésticas de las familias wichí nucleadas en diversas comunidades asentadas en la región (Trinchero y Belli, 2009), limitando el acceso a recursos que han sido históricamente vitales para la subsistencia en estas poblaciones.

En términos de trayectoria histórica, se puede señalar que para las primeras décadas de conformación del Estado Nacional, los pueblos indígenas del Gran Chaco, procuraron ser incorporados como mano de obra para el desarrollo de las actividades extractivas (Rosso, 2011). Sobre este proceso, también de avance y (re)definición de fronteras, estos pueblos fueron configurando prácticas de economías mixtas para la subsistencia, basadas en el trabajo asalariado precario y estacional en combinación con actividades fundadas en la caza y la recolección (Gordillo, 2006; Trinchero, 2014).

En el marco del nuevo avance de la frontera agraria -proceso que comienza de forma paulatina a partir de 1970 tomando un ritmo de crecimiento más acelerado a partir de 1990, y alcanzando niveles aún más inéditos en la década siguiente en relación a la tala de bosques (Shmithd, 2011) - el acceso al empleo temporal y estacionario disminuyó significativamente, debido a que el proceso de producción agrario actual, especialmente la producción intensiva de soja, requirió de menor mano de obra.

De esta forma, la caza, la pesca y la recolección, como prácticas que pervivieron en torno a una economía de subsistencia producida como estrategia adaptativa a los escenarios socio políticos planteados desde fines del XIX (Gordillo, 2006), se vieron fuertemente afectadas en este proceso, provocando una necesaria disminución de 
estas actividades. Del mismo modo, la actividad artesanal que contribuyó en las últimas décadas a sumar recursos monetarios a las economías familiares wichí, se vio también afectada por la pérdida de acceso a la madera (palo santo principalmente) y al chaguar (planta para uso alimentario y producción textil). La madera en las comunidades indígenas de la región representa no sólo un insumo para la producción artesanal, sino también un insumo para cocción de alimentos y calefacción.

Las políticas sociales (Asignación Universal por Hijo, Programa Pro Huerta para el desarrollo de agricultura familiar y becas Progresar entre las principales) mediante las cuales se procuró transferir recursos a estas poblaciones con intenciones de compensar los efectos de este proceso, no lograron atender la complejidad del impacto socio ambiental que conllevó la actividad extractiva desarrollada en las últimas décadas en la región chaqueña. Este proceso trajo aparejado el incremento significativo de problemáticas ligadas a la salud y el bienestar de estas poblaciones, complejizando las situaciones de riesgo. De esta forma, las bajas tasas de escolaridad que presentan los grupos étnicos de la región, se combinan con problemáticas vinculadas a la desnutrición y mal nutrición, parasitosis endémica, diabetes, adicciones y problemáticas vinculadas al uso de productos altamente tóxicos y contaminantes utilizados por las empresas agropecuarias para la producción. A este marco se suman situaciones críticas relacionadas con el acceso al agua y al agua potable, desatendida e invisibilizada aún como una de las mayores limitaciones que padecen comunidades indígenas y que condicionan las posibilidades mejoras que proponen algunos de estos programas.

Estos procesos agro productivos han ido desarticulando las estrategias y las prácticas de subsistencia ya mencionadas, tensionando la capacidad reproductiva y retentiva de las economías indígenas y campesinas de la región (Trinchero y Belli 2009). Como principal hipótesis de trabajo, comprendo que estas condiciones empujan a las poblaciones implicadas a producir necesariamente nuevas respuestas para confrontar las múltiples problemáticas, que son de orden material, como también de carácter simbólico ya que no sólo se requiere un apuntalamiento de las condiciones materiales, afectadas en este proceso, sino que el apuntalamiento de estas condiciones va a requerir de la incorporación y apropiación de saberes que circulan no sólo en los niveles primario y secundarios sino principalmente en los ámbitos de formación superior.

De acuerdo con esto, Rosso (2011:13) recalca que la articulación teórica entre las dinámicas económicas, materiales y simbólicas adquiere particular relevancia en los estudios que abordan la problemática intercultural en educación, si consideramos que las políticas educativas de carácter intercultural se caracterizan por un mayor abordaje de la dimensión cultural y lingüística, en detrimento de los aspectos económicos y productivos que indisociablemente permean a dichas dimensiones. Procurando avanzar con esta perspectiva y como se esboza en la introducción, las demandas por la formación superior (terciaria y universitaria) que se plantean en las comunidades wichí "Misión Chaqueña" y "Tierras Fiscales" y se analizan en este caso, están vinculadas a los procesos económicos y las (re)configuraciones territoriales que acontecen en el noreste de la provincia de Salta.

Profundizando en esta perspectiva analítica el territorio es comprendido como una instancia fundamental para la reproducción de los grupos sociales y como resultado de 
un proceso dinámico de producción/reproducción del "espacio social vivido". En este sentido, Haesbaert (2013:13) ofrece el concepto de "contención territorial, entendido como un proceso contemporáneo de las relaciones de poder referidas al espacio, donde se dibujan nuevas-viejas estrategias de control territorial". Este concepto es acuñado a partir de discutir el uso y abuso en las Ciencias Sociales sobre el concepto de "desterritorialización" al que se alude para dar cuenta del impacto que tienen ciertos procesos contemporáneos como el de las migraciones. En el caso de las migraciones puede verse de que manera a los procesos de desterritorialización se le contraponen procesos de (re)territorialización. Siguiendo esta línea, podemos considerar los procesos productivos que afectan la región noreste de Salta y el avance de la frontera agraria sobre los territorios indígenas y de pequeños productores, como procesos que tienden a la desterritorialización, en tanto que limitan las posibilidades de vida y reproducción del grupo, generando (re)configuraciones territoriales que implican a las dinámicas sociales, económicas, políticas y culturales de las comunidades wichí.

El estudio de caso muestra que las demandas por el acceso a la formación superior se tornan un elemento estratégico para la territorialización en las condiciones de vida contemporáneas. Para referirme al aspecto educativo, siguiendo a Bralich (2014), considero la educación como un proceso por el cual una generación busca comunicar conocimientos, técnicas y valores a las nuevas generaciones, con el fin de integrarlas fructíferamente al grupo social, considerando que todo grupo social con cierto grado de autonomía busca asumir su responsabilidad educativa y tomar sus propias decisiones. De esta forma, el énfasis respecto del rol de la educación, está puesto en la posibilidad de la reproducción social del grupo, es decir la "comunicación de la cultura" a las nuevas generaciones, preguntándome respecto de la relación dinámica entre reproducción y producción cultural, comprendiendo a la cultura en su dimensión dinámica e histórica, y entendiendo como señala Rockwell (2012:34) que "los sujetos transforman el sentido de los signos heredados y otorgan nuevos usos a esas herramientas culturales". De acuerdo con esto, es posible comprender que las demandas por acceder a la educación superior en las comunidades wichí que conforman este análisis, no suponen a priori una situación de asimilación o de imposición cultural "concluida", sino que por el contrario, se conforman en términos de apropiaciones y (re)significaciones de conocimientos y de saberes que se proponen ser integrados a la dinámica socio territorial de sus comunidades, para la producción y gestión de su espacio territorial. Al respecto, Emilio Fernández, wichí, Profesor de Matemática en el nivel medio, estudiante en la Universidad Nacional de Formosa y presidente de la Comisión Interétnica de Estudiantes de Pueblos Originarios (CIEPO) señala que la educación superior es hoy estratégica para la juventud indígena y el desarrollo de las comunidades, enfatizando que sin ella es impensable alcanzar "cambios sustanciales y significativos ni de instalar procesos de participación y el fortalecimiento de saberes en defensa de comunidades auto-sostenibles y autogestionables".

\section{Extensión universitaria, acceso a educación superior y procesos socio territoriales en comunidades wichí del Departamento de San Martin, Salta}


En el apartado anterior se puso el énfasis en el impacto socio ambiental producido en torno al desarrollo del modelo agroindustrial en el Gran Chaco Salteño, señalando que - la intensa actividad agroindustrial de las últimas décadas ha ido mermando la disponibilidad de recursos para la reproducción de estrategias grupales y familiares de subsistencia producidas por los grupos indígenas de la región en su experiencia histórica (Gordillo, 2006; Trinchero, 2001, 2007, 2013). Esto repercute en el acceso a recursos disponibles en el monte, la producción y consumo de alimentos a través de la caza, la pesca y la recolección, el acceso a maderas para la producción artesanal, la cocina y el trabajo asalariado, precarizado y estacionario.

Este contexto estimuló la conformación de reivindicaciones sociales vinculadas principalmente a la cuestión territorial y la problemática medio ambiental. En torno a estas luchas podemos ver en las comunidades indígenas de la región,(re)configuraciones sociales, políticas, económicas y territoriales que van resultando de la asociación entre diferentes actores comunitarios con ONG, fundaciones, instituciones del estado, instituciones religiosas, espacios políticos, educativos y universitarios donde se van desplegando nuevos y complejos entramados sociales (Mancinelli, 2014). En las últimas décadas, estos espacios institucionales han tenido una presencia importante en las comunidades indígenas, principalmente en lo que atañe a la promoción de proyectos de desarrollo rural o territorial. Los mismos buscan dar respuestas a las diversas problemáticas que se expresan en dichas poblaciones. En el caso de las universidades, la investigación se ha ido combinando con la extensión universitaria y los voluntariados produciendo experiencias particulares en las cuales se van reconfigurando ciertos aspectos de la relación entre "pueblos indígenas y Universidades".

Situándonos en el caso de la comunidad wichí Misión Chaqueña, encontramos instituciones como Unicef, desde la cual se impulsa entre otros proyectos, el desarrollo de huertas familiares. El proyecto de huertas se despliega en el marco de la promoción de la salud familiar y está especialmente orientado al cuidado de la infancia, que es el principal objetivo de esta institución. Quienes dentro de la comunidad, llegan a ser interpelados por esta institución para desarrollar el proyecto dentro de sus parcelas, acceden en muchos casos a aquellos insumos materiales que permiten dar impulso inicial a la creación de una huerta familiar. Es decir la presencia de estas instituciones implica, aunque de manera precaria, no sin tensiones, limitaciones y contradicciones, el acceso y circulación de bienes materiales y simbólicos. En el caso del desarrollo de huertas, estos bienes van a ser principalmente mangueras para riego, bombas de agua, insumos para perforación de pozos de agua, cable para conectar la bomba, y semillas. Si el proyecto se desarrolla con cierto éxito se accede a alimentos vegetales de estación: hortalizas como tomates, calabaza y maíz, entre los principales productos. De esta manera participar del proyecto, representa no solo producir una huerta sino también alcanzar algunas mejoras de infraestructura en la unidad familiar, como lo son la bomba y el pozo para el agua comprendiendo el agua en estas poblaciones como un recurso de acceso complejo y muy disputado.

Las entrevistas realizadas a los jóvenes que participan de estas propuestas, remarcan los desafíos que conlleva sostener una huerta en esta región, donde las dificultades para acceder al agua se combinan con la falta de conocimientos sobre el riego, y conocimientos específicos vinculados a los cultivos estacionales. Respecto del 
programa nacional del INTA, particularmente, las entrevistas señalan que en términos generales, estos programas se limitan a la entrega de semillas pero no responden a las demandas de capacitaciones que se requieren para el desarrollo de la agricultura familiar, ni consideran las limitaciones vinculadas a la infraestructura. Algunos jóvenes recorren los $50 \mathrm{~km}$ a la ciudad de Embarcación para acceder a una computadora y una red de internet con el objetivo de conseguir información sobre agricultura.

Estas entrevistas permiten analizar reclamos educativos vinculados con la formación secundaria donde la modalidad agrotécnica es una de las más demandas por las comunidades indígenas de la región, mostrando una fuerte relación entre educación y formación para para la producción y la reproducción de la vida individual y familiar.

Lo que interesa rescatar de las entrevistas para el análisis que propongo aquí, no son las limitaciones que tienen estos programas, sino cómo estas limitaciones se vinculan en procesos de conformación de demandas educativas que van interpelando a instituciones educativas, fundaciones, ONGs y organizaciones sociales y políticas.

Desde este encuadre, es posible pensar las políticas y los programas de extensión universitaria que se emplazan en las comunidades indígenas de la región. De acuerdo con lo planteando en el párrafo anterior, comprendo que también desde "la universidad"iii se han ido configurando y moldeando diferentes tipos de intervenciones con relación al impacto producido por los procesos políticos, económicos y culturales de las últimas décadas. Esto se conjuga incorporándose con las particularidades del proceso de (re)configuración socio territorial, y van funcionando también como un estructurante en las demandas educativas de formación terciaria y universitaria.

El trabajo etnográfico de Ossola (2014) ofrece un ejemplo interesante al indagar en el proceso de adaptación de un grupo de jóvenes wichí en la Universidad Nacional de Salta (UNSa). En este trabajo se remarca como un hito en la trayectoria escolar y de vida de los Jóvenes universitarios wichí, la experiencia vivida como estudiantes del colegio secundario de su comunidadiv y el encuentro con un equipo de extensión universitaria de la UNSa, mostrando de qué manera esta experiencia modificó los horizontes escolares de estos jóvenes, motivándolos a ingresar a la universidad y colaborando para generar condiciones de ingreso. La autora caracteriza este tipo de ingreso como un "ingreso mediado" porque el mismo es estimulado por los docentes que participaron del proyecto de extensión, ayudando a construir respuestas tanto del orden simbólico como material, mostrando un ingreso a la universidad que podemos decir, sigue una lógica distinta a la que se provee institucionalmente. El trabajo de Ossola, da cuenta también de cómo se modifican las perspectivas de los docentes universitarios del programa de extensión respecto de las condiciones de igualdad socioeducativa, estimulando la conformación de iniciativas a nivel institucional para responder a las necesidades concretas de los grupos indígenas y también da cuenta del modo en el regreso de los jóvenes universitarios a la comunidad modifica las relaciones intergeneracionales en la misma.

De manera similar podemos referirnos a la experiencia de inclusión de jóvenes wichí en la Facultad de Ciencias de la Salud de la Universidad de Mar del Plata. En este caso se puede apreciar una trayectoria en algunos aspectos similar al caso trabajado por Ossola, en lo que refiere principalmente a lo que la autora define como "ingreso mediado" ya que la inclusión de estos jóvenes se produce a partir de una 
experiencia de trabajo de extensión universitaria. En este caso el equipo de extensión está conformado por docentes y estudiantes de la Licenciatura en Enfermería de la Facultad de Ciencias de la Salud de la Universidad Nacional de Mar del Plata. Este equipo viene trabajando hace 5 años en las comunidades wichí del Municipio de Embarcación, con la perspectiva de producir respuestas a las problemáticas de salud que se expresan en estas poblaciones, principalmente la parasitosis endémica, la salud reproductiva, la tuberculosis, y la alimentación en niños y adultos.

Luego de algunos años y de un trabajo centrado principalmente en la prospección de campo, el equipo va configurando sus actividades de transferencia a través de un curso para formar Promotores de Salud. El curso se diseñó no solo para capacitar a jóvenes y adultos para que ocupen "roles estratégicos en el cuidado de la salud en sus comunidades" buscando el empoderamiento de estas comunidades, sino que en función de este empoderamiento, el proyecto también se diseña con el objetivo de otorgar certificaciones que permitan acceder a un trabajo asalariado. Si bien el curso se dictó en una sola de las comunidades con las que trabaja este equipo, convocó a jóvenes de las distintas comunidades. Durante esta experiencia formativa el equipo de extensión se planteó la posibilidad de que la formación de algunos de estos jóvenes alcance un nivel universitario. Las entrevistas a los jóvenes que realizaron el curso de promotores de salud mostraron que el curso tuvo una importante repercusión ya que cubría las expectativas de continuidad en sus trayectorias escolares, principalmente en función de conseguir una formación orientada al trabajo asalariado. Cabe advertir que la falta de recursos económicos u obligaciones de trabajo en las unidades domésticas familiares y el secundario inconcluso son las principales causas que limitan la continuidad en el nivel superior esbozadas por los jóvenes durante las entrevistas. La percepción del equipo de salud sobre el escaso acceso que tienen estos jóvenes a la formación profesional de nivel superior motivó al equipo a colaborar con algunos jóvenes, que cumplían con el requisito de secundario terminado, a ingresar a la Universidad de Mar del Plata. De esta forma, en el 2014 siete jóvenes de estas comunidades, viajaron a Mar del Plata con el apoyo del equipo de extensión universitaria, la ayuda familiar y algunos insumos que fueron solicitados al municipio para estudiar en dicha universidad. La experiencia del 2014 llevó a que durante 2015 se incorporara un estudiante más.

El apoyo que brindó el equipo de extensión consistió en ayuda económica para pasajes, cuyos fondos fueron de los particulares y no involucró presupuesto de la universidad. Asimismo se trabajó la integración al ámbito universitario en las primeras etapas de ingreso, aconsejando el recorrido de materias en las cuales inscribirse e informarlos de las principales dinámicas que hacen a la cursada. El equipo también procuró acompañar la gestión de las becas de apuntes y el refrigerio que brinda la universidad a todo estudiante que lo requiera por medio de la secretaria de Bienestar Universitario. Un momento crítico de ese proceso fue el alojamiento, ya que no se cuenta con recursos económicos para tal fin. Finalmente se gestiona mediante la solidaridad del dueño de un hostal que pone habitaciones a disposición de los jóvenes wichí universitarios en temporada de poco turismo la cual coincide con el periodo lectivo.

Por su parte, el municipio de Embarcación, mediante cartas de solicitud, colabora con el pago de algunos de los pasajes en el tramo Embarcación/Buenos Aires ya que 
no cuentan con servicio directo a la Ciudad de Mar del Plata. En relación al apoyo que van recibiendo de sus familiares para sostenerse en esta ciudad, el mismo proviene principalmente de la producción artesanal que mediante encomiendas en ocasiones hacen llegar para que los estudiantes vendan en la misma facultad.

El trabajo de campo realizado en la Universidad de Mar del Plata muestra que este grupo de jóvenes encontró en la ciudad, en su condición de wichí universitarios, la posibilidad de ampliar el apoyo recibido por el equipo de Extensión y de sus familiares, sumando el acompañamiento afectivo y material de otros actores de la comunidad educativa de esta institución, como los asistentes sociales (que atendieron sus pedidos de becas), los tutores pedagógicos (que dispone la facultad para todo estudiante que los requiera), los compañeros de estudios y los docentes que progresivamente fueron visibilizándolos como estudiantes wichí.

Cabe remarcar que el ingreso de estos jóvenes se produce principalmente por mediación del Equipo de extensión y como una política de la Universidad por lo cual en muchos espacios no son necesariamente reconocidos como estudiantes indígenas.

Puede verse que este apoyo que van recibiendo los estudiantes universitarios wichí se conforma como una red social de contención a la que apelan para ir resolviendo otras necesidades, como lo es el dinero para pasajes de retorno a las comunidades al finalizar el ciclo lectivo, tarjetas para celulares para mantener la comunicación con sus familias y los pasajes en colectivo. La experiencia cuenta ya con tres años de trayectoria en la cual algunos de estos jóvenes han comenzado a insertarse en el mercado laboral mediante changas que involucran el cuidado de personas mayores, es decir trabajos vinculados a su práctica profesional y en otros casos en la oferta gastronómica.

Con relación a las tutorías, que como señalamos no responden a un acompañamiento fundado en el reconocimiento de la identidad étnica, las entrevistas muestran que en pocas ocasiones estas han sido escogidas por los estudiantes wichí para resolver las dificultades que tienen para acomodarse a los ritmos y los contenidos académicos en las diferentes materias, expresando más afinidad con la ayuda ofrecida por compañeros de clases y en la participación en grupos de estudio.

El sistema de becas que otorga la universidad a todo estudiante que requiera de éste ha podido complementarse, en el caso de los jóvenes menores de 24 años, con las prestaciones sociales que ofrece el gobierno nacional (Plan Progresar y Becas Estimulo para jóvenes de 18-24 años).

En términos muy generales podemos referirnos a la cuestión étnico/identitaria como una variable fundamental en la constitución de estas redes de ayudas que fueron descriptas en este apartado. Puede decirse que el reconocimiento de su identidad étnica se vincula no solo a cuestiones culturales sino también se las asocia a las condiciones de desigualdad social y económica que atraviesa a estos pueblos.

Para referirme a un caso más que permite profundizar y articular aspectos mencionados en los casos anteriores, se puede mencionar a la experiencia de extensión universitaria que realiza en Misión Chaqueña un equipo de la Facultad de Agronomía de la UBA, desde la cual se colaboró para la producción de una Huerta Comunitaria. En este caso se trata de un proyecto UBANEX ${ }^{v}$ realizó las tareas de instalación de la Huerta, y proveyó los insumos más importantes que esta requería. Puede decirse que estas experiencias responden a demandas de formación en 
materia de agricultura, que se plantean actualmente en estas comunidades como parte de la búsqueda y producción de nuevos recursos para la subsistencia y gestión del espacio territorial. Durante los trabajos de campo, pude ver cómo la demanda vinculada a conocimientos para la producción de agricultura familiar es tomada por diferentes familias de esta comunidad y pasa a ser también gestión de la junta vecinal $^{\mathrm{vi}}$. Estos proyectos en la práctica van convergiendo con algunas políticas sociales. En este caso podemos referirnos al INTA desde donde se promueve la agriculturización como forma de paliar algunas problemáticas, principalmente Alimentarias. En relación al programa del INTA las entrevistas señalan que el mismo, se limita a proveer semillas no asumiendo las necesidades de capacitaciones y sin contemplar las problemáticas de infraestructura que se plantean en la comunidad. En este marco entro la junta vecinal demanda que la escuela secundaria que está dentro de la misma se vuelque a la modalidad agro técnica.

En este caso, desviándonos un poco del eje central de este artículo, cabe mencionarse algunos aspectos en torno al edificio del colegio secundario. El secundario de Misión Chaqueña abre en los años 2000 como anexo del secundario que se encuentra a $15 \mathrm{~km}$, en el pueblo de Pedro Lozano y pocos años después se independiza. Hasta el 2015 funciona a contra turno de la escuela primaria. Para 2014/2015 se concreta la construcción del edificio propio del colegio secundario.

Si bien el municipio de Embarcación dispuso la apertura del nuevo edifico del secundario para principios del 2015 con ciertas obras terminadas, la junta vecinal se opuso a esta apertura hasta que se concretara principalmente la obra hidráulica planteada en el proyecto. Esta obra consistía en la perforación de un pozo para la extracción de agua menos contaminada, es decir a una profundidad de por lo menos 40 metros y la compra de la bomba extractora. Como ya mencionamos el agua se torna en un recurso estratégico siendo una de las principales limitaciones que enfrentan para el desarrollo de huertas familiares y comunitarias, porque es un recurso escaso y porque las napas más superficiales posen un alto grado de contaminación. El crecimiento demográfico de la comunidad junto a la falta de mantenimiento de las obras realizadas décadas atrás, acentúan la problemática del acceso a este recurso.

La municipalidad disponía la apertura de la escuela, planteando como solución provisoria la utilización de la bomba y el pozo cercanos al colegio. La comunidad se resistió a la apertura del colegio en dichas condiciones ya que esto implicaba una merma importante del agua para las casas aledañas al colegio. Asimismo puede decirse que la negociación de este conflicto se conformaba como estrategia para avanzar en las demandas de infraestructura de la comunidad en general, ya que la escuela representó también la posibilidad de sumar una bomba y acceso a agua de mejor calidad. En este escenario, la construcción de la huerta dispuesta por el equipo de extensión de agronomía quedó a medio terminar y a la espera de que se concretaran las obras.

Para agosto de 2015 pese a que no se resuelven estas condiciones de infraestructura, Misión Chaqueña accede a la apertura del edificio. Esta renegociación se vincula con la posibilidad de ampliar la oferta educativa en la comunidad hacia el nivel superior con la apertura de los Institutos de Formación Docente en la modalidad de Educación Intercultural Bilingüe que viene siendo una de las principales políticas educativas a nivel superior en la provincia de Salta desde 2011. Las entrevistas 
muestran que la comunidad tiene dos expectativas centrales en la apertura de una sede de estos profesorados en el territorio. Una de ellas refiere a la necesidad de contar con docentes propios, que compartan los horizontes de desarrollo de la comunidad y que confíen en las capacidades intelectuales de sus jóvenes que muchas veces son subestimadas por los docentes que son externos a la comunidad y que no comparten adscripción étnica ${ }^{\text {vii }}$. Otra expectativa refiere a la posibilidad de que los jóvenes que no cuentan con acceso a trabajo encuentren en este espacio una actividad ${ }^{\text {viii. }}$.

Me permito sumar a estas experiencias que presento aquí, la propia actividad que me encuentro realizando como becaria de la UBA, la cual no está exenta de decisiones institucionales e individuales que interpelan a la temática de la relación entre universidad y pueblos indígenas. La misma investigación es resultado de mi participación en un equipo de extensión con trayectoria de trabajo con estas comunidades. Como señalan Trinchero y Petz (2014), las vinculación entre la universidad y el territorio tiene su propia trayectoria, y podemos situarla a partir de la reforma de 1918 cuando las luchas estudiantiles de la época pusieron en cuestión el rol de la universidad y la necesidad de extender la presencia de la universidad en la sociedad. Sin embargo en las últimas décadas, la creación de espacios nuevos que consideran los entramados locales, y las políticas de financiamiento que promueven proyectos que procuran una vinculación con los procesos territoriales y las políticas de integración social, modifican los términos de esta relación donde cada vez más se articula el trabajo con las organizaciones sociales que Trinchero y Petz definen como "territorialización de la universidad" (ob.cit.:143).

En este apartado se procuró ofrecer una descripción de diferentes experiencias que permiten acercarnos a diversos modos de vinculación que se van construyendo entre espacios universitarios y pueblos indígenas y los efectos o sinergias que estas experiencias producen. Una de las primeras conclusiones que pueden proponerse de este apartado es que en estas experiencias vemos formas particulares en que circulan "saberes" que se producen en los espacios universitarios. En este caso podemos referimos al programa de promotores de Salud de la Universidad de Mar del Plata, y al programa de extensión de la Facultad de Agronomía donde el trabajo de campo dejó ver transferencias de saberes que en la praxis se vinculan con la atención de salud y con el desarrollo de la agricultura familiar y comunitaria. Al mismo tiempo puede verse que la presencia tanto de los programas de extensión universitaria como las propuestas de políticas sociales como la del INTA que citamos aquí, generan nuevas demandas educativas no sólo sobre el nivel superior sino también en articulación con el nivel medio. Como se mencionó la expectativa de la comunidad es que el secundario se inscriban en la modalidad agro técnica, por su parte el programa de extensión de la Universidad de Mar del Plata a partir del curso de promotores y el acompañamiento de jóvenes para el ingreso a la carrera de enfermería en dicha universidad, estimuló a muchos jóvenes a culminar los estudios secundarios que fueron abandonado en su momento.

\section{Consideraciones finales}


Para cerrar este trabajo interesa remarcar el modo en que comprendo que estas experiencias permiten re-pensar la relación entre las instituciones educativas y la comunidad, mostrando que estas no sólo están instaladas en la comunidad sino que conforman también procesos espaciales y territoriales. Aguilera Urquiza y Nascimiento (2010) tomando los aportes de la geografía crítica, asumen el territorio como un producto que resulta del "esfuerzo colectivo de los grupos sociales para ocupar, usar, controlar e identificar el territorio con determinada parcela del ambiente físico" (Little, 2002 en Ob.cit:7) y donde todo proceso de territorialización implica una apropiación y producción y usufructo de recursos materiales y simbólicos (Milton Santos, 2000).

De acuerdo a las experiencias someramente abordadas en este trabajo, vinculadas con los procesos territoriales que se producen en la región del gran chaco salteño, se puede comprender de qué manera la apropiación de recursos simbólicos producidos en los espacios de enseñanza superior se torna clave para la producción del espacio territorial en estas comunidades, mediante los cuales se procura alcanzar ciertos niveles de bienestar en la comunidad.

Siguiendo con esta perspectiva entiendo que recuperar la riqueza de los debates en torno a la dimensión territorial y espacial, que se producen en el campo de la antropología económica, y de la geografía crítica permiten sumar el análisis espacial a los procesos educativos, ampliando la comprensión respecto de las tensiones y las dinámicas que se producen en los espacios socio territoriales, donde los procesos educativos adquieren sus particularidades acercándonos a una comprensión de los sentidos y las representaciones que la educación media y superior asumen en estos contextos inter-étnicos. Parafraseando a Haan y Elbers (2005), "la diversidad debe comprenderse como resultado del hecho de que las herencias culturales, y las relaciones de poder adoptan nuevas formas a medida que ambas se reconfiguran en las condiciones locales, y se conforman unas a otras en el aquí y el ahora".

En esta dirección, Ossola (2013) recupera la noción de frontera como posibilidad heurística para analizar las experiencias que se realizan en los espacios escolares, desde la cual se puede observar la heterogeneidad de sentidos que se producen en diferentes contextos, y donde la frontera pasa a concebirse no ya como un límite sino como un espacio de flujos e intercambios (Trinchero 2014). En este proceso puede comprenderse el eje espacial, de manera dinámica, ya que la categoría de frontera permite re-pensar el espacio como algo dado, para concebírselo como algo producido por actividad social. Schmidt (2014) señala que en las ciencias sociales el eje temporal para el análisis de los procesos se ha privilegiado en detrimento del eje espacial, debiéndose principalmente a la forma en que las ciencias sociales y en especial las políticas públicas han comprendido el espacio y el territorio. Siguiendo este planteo, recuperamos a Segato (2007) quien advierte que las políticas públicas siguen aún permeadas por la perspectiva estado céntrica, donde el territorio y estado se perciben de manera análoga negando la presencia de otros espacios y las diversas formas de comprender, vivir y experimentar el territorio, las cuales entendemos aquí, entran en tensión en los procesos sociales concretos.

Puede decirse que estas perspectivas estado-céntricas respecto del territorio trascienden a las políticas educativas que se vienen realizando en pos de mejorar indicadores de inclusión y de calidad educativa en poblaciones étnicamente definidas, preguntándome de qué manera la etnografía educativa puede colaborar para irrumpir 
estas perspectivas. En esta dirección Corbetta postula el enfoque del territorio como una posibilidad concreta para repensar la política educativa pública en general (2009:264).

Como se sugiere en el título de este trabajo, la educación superior puede comprenderse en este contexto como un recurso estratégico para el desarrollo de la comunidad en las condiciones actuales donde, producto de la desforestación y contaminación ambiental, se ha producido una histórica merma de los bosques que proveyeron a muchas generaciones de "recursos" necesarios para la subsistencia. Debe comprenderse también, la educación como un recurso en disputa en tanto que el acceso esta mediado principalmente por la lucha histórica que vienen dando los pueblos indígenas para el acceso y la inclusión a la formación universitaria a la que se suma la disputa por recursos para la producción de conocimientos y de espacios académicos de nivel superior que se configuren para alcanzar mejores condiciones de vida que las que se viven en el presente histórico.

De acuerdo con esta perspectiva podemos (re)situar la mirada de los procesos educativos que suceden en el territorio entendido como lugar empírico y dado, para (re)pensarlos como espacios que coadyuvan en los procesos de producción territorial. Así, la educación formal y universitaria emplazada en las comunidades wichí mediante proyectos de extensión universitaria, a través de complejos procesos de apropiación cultural deviene en un mecanismo no sólo de defensa y de revalorización cultural de la identidad indígena (Ossola, 2013) sino también del espacio socio territorial y la posibilidad de recuperar dinámicas sociales para el sostenimiento de la autonomía relativa de las comunidades wichí del Gran Chaco.

\section{Bibliografía}

Aguilera Urquiza A. H. y Nacsimento A.C. (2010) "O desafío da interculturalidad na formação de professores Indígenas”. Espaço Ameríndio, Porto Alegre, v. 4, n. 1, p. 44-60, jan./jun.

Bralich, J. (2014). "Qué es la Educación Latinoamericana". En Ideas de la Educación Latinoamericana, tomo 1, Un Balance Historiográfico. Nicolás Arata y Miriam Southwell (comps.), 93-102

Corbetta, S. (2009). Territorio y educación. La escuela desde un enfoque de territorio en políticas públicas. En N. López De relaciones, actores y territorios: hacía nuevas políticas en torno a la educación en América Latina (263-304). Buenos Aires: UNESCO-IIEP.

Fernandez, Emilio (2016).Proceso de inserción y participación de jóvenes indígenas en la Educación Superior en Formosa. Emilio Fernández -UNFormosa -Argentina. En http://untref.edu.ar/sitios/ciea/wpcontent/uploads/sites/6/2015/05/Compilado-ponencias.pdf

Gordillo, G. (2006). "En el Gran Chaco. Antropologías e Historias". Prometeo libros. Bs As.

Haesbaert, R. (2013). Del mito de la desterritorialización a la multiterritorialidad, Cultura y representaciones sociales 8 (15): 9-42.

Haan, M., y Elbers, E. (2005). Reconfigurando la diversidad en un salón de clases local: asuntos de comunicación e identidad en escuelas multiculturales en Holanda. 
Leavy, P. (2014). La lógica del cuidado en estrategias sanitarias destinadas a la población Materno infantil. Século xxi, revista de Ciências Sociais, v.4, no 2, p.242-268, jul./dez. 2014 issn: 2236-6725.

Mancinelli, G. (2012). Educación como "recurso" en contextos de disputas territoriales en las poblaciones Wichí y Guaraní. pp 31-44. Revista Argentina de Economía y Ciencias Sociales Vol XIV, № 19, Invierno, Ediciones de la Universidad. Buenos Aires - Argentina.

Mancinelli, G. (2014). "Sentidos y perspectivas en las demandas de Educación Superior en las comunidades Wichí. Pensando antecedentes y trayectorias históricas." XI Congreso Argentino de Antropología Social. Rosario, 23 al 26 de Julio de 2014.

Marcus, G. (2001) "Etnografía en/del sistema mundo. El surgimiento de la etnografía multilocal". Revista Alteridades, 11 (22). pp. 111 a 127.

Mato, D. (2014) Educación superior y pueblos indígenas en américa latina. Contextos, experiencias y desafíos. Revista Uturunku Achachi (Vol.3), pp.109-128 Recuperado de: uturunkuachachi.academialatinoamericana.org.

Milton, S. (2000). La naturaleza del espacio. Técnica y tiempo. Razón y emoción. Barcelona. Ariel S. A

Ossola, M. M. (2013). Jóvenes indígenas en la frontera: relaciones entre etnicidad, escolaridad y territorialidad. Revista Latinoamericana de Ciencias Sociales, Niñez y Juventud, 11 (2), pp. 547-562.

Ossola, M. M. (2014). Aprender de las dos ciencias. Etnografía con jóvenes wichí en la educación superior de Salta. Santiago del Estero: EDUNSE.

Paladino, M. (2009) "Pueblos indígenas y educación superior en la Argentina. Datos para el debate". En ISEES: Inclusión Social y Equidad en la Educación Superior, Núm. 6, 81-122.

Rockwell, E. (2010). "Tres planos para el estudio de las culturas escolares". En: Aprendizaje y contexto: contribuciones para un debate. N. E. Elichiry, Coord. Buenos Aires: Manantial.

Rosso, L. (2011) "Educación estatal para indígenas del Chaco Argentino: tensiones históricas". En: Paladino, Mariana - García, Stella Maris (Coord.). La Escolarización en los Pueblos Indígenas Americanos. Impactos y desafíos; Ecuador: Abya Yala, ISBN: 978-9978-22-963-7, pp.35-61.

Rotman M., Radovich J. C., Balazote, A. (2007) "Las producciones Artesanales de los pueblos indígenas en contextos de relaciones interétnicas" En: Pueblos originarios y problemática artesanal: Procesos productivos y de comercialización en agrupaciones Mapuches, Guaraní/ Chané, Wichí, Qom / Tobas y Mocovíes. Editores: Universidad Nacional de Córdoba. Centro de estudios Avanzados. Conicet. Córdoba Argentina.

Segato R. (2007). "Identidades políticas/alteridades históricas: una crítica a las certezas del pluralismo global". En R. Segato La Nación y sus otros. Raza, etnicidad y diversidad religiosa en tiempos de política de la identidad (pp. 3770). Buenos Aires: Prometeo.

Schmidt M. (2011). "Pueblos Indígenas, Estado y Territorio en tiempos interculturales en la provincia de salta". Estudios Socioterritoriales. Revista de Geografía. № 10. PP. $13-4013$.

Trinchero H. y Belli E. (2009). Introducción. Trinchero, Hugo y Elena Belli, (coord.). En: Fronteras del desarrollo. Impacto social y económico. Biblos.

Trinchero H., Campos Muños, L., Valverde S. (2014). Perspectivas antropológicas para el análisis histórico de las fronteras. En Trichero, H. et al. (comps.); Pueblos indígenas, estados nacionales y fronteras. Tensiones y paradojas de los procesos de transición contemporáneos en América Latina I: 27-71. 
Trinchero H. H. y Petz I. (2014) "La cuestión de la territorialización en las dinámicas de integración universidad- sociedad. Aportes para un debate sobre el "academicismo". En: Papeles de Trabajo № 27 - Julio 2014 -ISSN 1852-4508 Centro de Estudios Interdisciplinarios en Etnolingüística y Antropología SocioCultural 142

\section{Notas:}

'El material producido para este análisis, forma parte de los trabajos de campo que me encuentro realizando para la tesis Doctoral: Educación superior en contextos interculturales. Un estudio acerca de las demandas educativas de las comunidades wichí y guaraníes de Salta", Con dirección del Dr. Alejandro Balazote y codirección de la Dra. Ana Carolina Hecht en el marco del Proyecto UBACyT, "Impacto del conflicto por los recursos naturales del modelo extractivo en poblaciones étnicamente definidas", con sede de trabajo en la Sección de Antropología Social del Instituto de Ciencias Antropológicas de la Facultad de Filosofía y Letras de esa casa de estudios.

ii Dicho de otro modo, las nuevas condiciones que impone el actual modelo productivo y extractivo ha quebrantado sus estrategias y patrones alimentarios,- sus gramáticas culinarias-, perturbando los criterios a partir de las cuales lograban una dieta que podía considerarse más eficiente, proceso que conlleva al mismo tiempo, a una pérdida importante de autonomía por parte de estos grupos ya que se incrementa la demandas de productos provenientes del mercado. Estas condiciones empujan a las poblaciones afectadas a incorporar, modificar y resignificar recursos materiales y simbólicos.

iii Nos referimos a "la Universidad" entre comillas como un conjunto que involucra a las diversas universidades, reconociendo este concepto como un constructo y entendiendo que al interior de este conjunto advertimos diferentes marcos institucionales. Si bien este aspecto no es profundizado en el presente trabajo, se desprende del trabajo de campo que los distintos espacios académicos mencionados tienen perspectivas propias para pensar la intervención y el acompañamiento de las comunidades indígenas.

iv Los Troncos es el nombre ficticio que la autora da a la comunidad wichí de la provincia de Salta a fin de resguardar la privacidad de los actores interpelados para la investigación.

$\checkmark$ UBANEX es un Programa de Subsidios para proyectos de Extensión Universitaria de la Universidad de Buenos Aires cuyo objetivo es fortalecer las políticas de extensión hacia el interior de la Universidad; lograr la articulación entre diversos proyectos y programas buscando unificar esfuerzos, y al mismo tiempo, compartir experiencias y datos, evitando la dispersión de recursos y la duplicación de dispositivos; jerarquizar la tarea docente en el marco de las acciones de extensión universitaria, valorando el impacto en el currículo y las acciones pedagógicas que resinifiquen la práctica social; propiciar un nivel alto de participación de estudiantes, fomentando además la interdisciplinariedad.

vi Las comunidades indígenas han ido modificando las formas de representación y participación política intracomunitarias a fin de ir resolviendo modos de vinculación con el estado en los nuevos contextos sociales e históricos y en relación a los nuevos marcos legales que dan reconocimiento a los derechos de estos pueblos. En el caso de estas dos comunidades wichí, la organización política articula diferentes instancias de reconocimiento de autoridades, una de ellas es la figura del Cacique vinculada a un sistema hereditario que continúa cumpliendo algunas funciones estratégicas en la organización social de estas comunidades. La junta vecinal, a diferencia de la figura del cacique, es elegida cada dos años mediante asamblea y voto de todos los miembros de la comunidad. Sus funciones se vinculan a la gestión de áreas estratégicas para la comunidad, como la educación, el agua, la salud y el reconocimiento jurídico del territorio.

vii Entrevistas realizadas en abril de 2015 a delegados de la junta vecinal de la comunidad wichí Misión Chaqueña.

viii Esta perspectiva coincide con la planteada por dirigentes collas, de la provincia de Jujuy durante la audiencia pública que se realizó en agosto de 2013 en el Congreso Nacional con el fin de discutir el proyecto de ley para la creación de una Universidad Nacional Intercultural de 
los Pueblos Indígenas quienes defendieron el proyecto en el marco de la producción de políticas para la juventud indígena (Lenton et. al, 2015). 